\title{
A utilização do Método de Rorschach no diagnóstico diferencial da Esquizofrenia e Transtorno Dissociativo de Identidade*
}

\author{
Marcello de Abreu Faria ${ }^{1}$, Euthymia Brandão de Almeida Prado ${ }^{2}$, Wânia Cristina de Souza ${ }^{1}$, \\ Vaneila Ferreira Martins ${ }^{3}, \&$ Vania Moraes Ferreira ${ }^{1, * *}$ \\ ${ }^{1}$ Universidade de Brasília, Brasília, DF, Brasil \\ ${ }^{2}$ Universidade Federal de São Paulo (UNIFESP), São Paulo, SP, Brasil \\ ${ }^{3}$ Universidade Federal de Goiás, Goiânia, GO, Brasil
}

\begin{abstract}
RESUMO - o objetivo deste artigo foi investigar a prevalência do Transtorno Dissociativo de Identidade (TDI) em dez pacientes diagnosticados com esquizofrenia com característica paranóide, com base no Método de Rorschach. Foram registrados indicadores ou sintomas psicológicos que caracterizaram o diagnóstico diferencial desses pacientes, analisadas diferenças clínicas específicas e verificado o enquadramento dos dados obtidos, com a aplicação da Técnica de Rorschach. Para fins de diagnóstico diferencial, observou-se que dois pacientes apresentaram sintomas diferenciais aos da esquizofrenia, sendo que um deles apresentou sintomas dissociativos, não sendo confirmada a prevalência do TDI entre os esquizofrênicos. No caso da esquizofrenia e do TDI, devido às características similares que apresentam, avaliações multidisciplinares são indicadas para se fazer o diagnóstico diferencial entre eles.
\end{abstract}

PALAVRAS-CHAVE: esquizofrenia, transtorno dissociativo de identidade, trauma, rorschach

\section{Using the Rorschach Method in The Differential Diagnosis of Schizophrenia and Dissociative Identity Disorder}

\begin{abstract}
The aim of this article was to investigate the prevalence of the Dissociative Identity Disorder (DID) in ten patients diagnosed with schizophrenia with paranoid characteristic, based on the Rorschach approach. Psychological symptoms that characterize the differential diagnosis of these patients were registered, specific clinical differences were analyzed and the framework of the data obtained with the application of the Rorschach approach. For purposes of differential diagnosis, it was observed that two patients had differential symptoms to schizophrenia, one of which showed dissociative symptoms, not confirmed the prevalence of DID among schizophrenics. In the case of schizophrenia and DID due to exhibit similar characteristics, multidisciplinary evaluations are set to make a differential diagnosis between them.

KEYWORDS: schizophrenia, dissociative identity disorder, trauma, rorschach
\end{abstract}

A esquizofrenia é uma síndrome prevalente entre as desordens mentais, com status de transtorno psiquiátrico desafiador e muito grave (Asher, Fekadu, \& Hanlon, 2018; Vita \& Barlati, 2018). É um distúrbio psicótico caracterizado pela dissociação das funções psíquicas, e seus fenômenos de maior relevância são os distúrbios de relacionamento e de afeto, a desorganização do pensamento, o impedimento para delimitar a realidade e a fantasia, variadas manifestações do comportamento e incapacitação progressiva, cujos déficits começam a se manifestar ainda na infância ou na adolescência, percebidos antes dos sintomas psicóticos. Entre as características que mais se destacam na esquizofrenia estão os delírios, transtornos de pensamento e de fala, perturbação das emoções e avolição (Finsaas, Bufferd, Dougherty, Carlson, \& Klein, 2018; Salamon, Santelmann, Franklin, \& Baethge, 2018; Vita \& Barlati, 2018).

Pela teoria genética, a esquizofrenia é uma desordem hereditária. Entre os sintomas somáticos, sobressaem-se

* Apoio: $\mathrm{CNPq}$

**E-mail: vmmf@unb.br

n Submetido: 06/09/2016; Revisado: 25/10/2016; Aceito: 04/04/2017. 
os déficits sensoriais adquiridos ao longo da vida, que geralmente resultam em percepções distorcidas e geram vácuos sensoriais. Pela $5^{\mathrm{a}}$ edição do Manual Diagnóstico Estatístico de Transtornos Mentais (DSM-V; American Psychiatric Association [APA], 2014), o espectro da esquizofrenia inclui transtornos psicóticos e transtorno da personalidade esquizotípica (Cordeiro, Silva, \& Vallada, 2012; Howes, McCutcheon, Owen, \& Murray, 2016; Kendler, 2016; Ma, Gu, Huo, Li, \& Luo, 2018).

O Transtorno Dissociativo de Identidade (TDI), popularmente chamado de "personalidade múltipla", envolve a percepção, um dos elementos essenciais de sua análise para indicar a possibilidade do controle racional do indivíduo sobre os afetos e as emoções. É caracterizado por perspectivas discrepantes de seus subsistemas, como partes dissociadas da identidade, personalidades alternativas ou estados alterados de consciência, tendo barreiras psicobiológicas limitantes. Cada personalidade pode ser vivenciada como se tivesse uma história pessoal distinta e própria, autoimagem e identidade própria, inclusive nomes diferentes; cada uma determina comportamentos, atitudes e sentimentos diferentes (Ribáry, Lajtai, Demetrovics, \& Maraz, 2017; Schimmenti, 2017).

Como há um fracasso psíquico na integração de aspectos da identidade, da memória e da consciência, quando o indivíduo expressa uma personalidade, há amnésia em relação à outra. Entre os sinais e sintomas, há: histórico familiar de transtornos dissociativos, lançando evidências de que o TDI é transgeracional. Não são raros os sintomas somáticos associados, incluindo convulsão. Pelo DSM-5 (2014), nesse transtorno há lacunas recorrentes na recordação de eventos cotidianos ou incapacidade de relembrar informações pessoais (não explicadas por esquecimento normal); prejuízo no funcionamento social, profissional ou em outras esferas importantes da vida (Braun, 1990; Dorahy et al., 2014; Gentile \& Dillon, 2013; Leonard \& Tiller, 2016).

Historicamente, a esquizofrenia é confundida com desordens dissociativas, possivelmente pela disfunção neuropsicológica observada nos respectivos portadores, além de seus aspectos conceituais: a esquizofrenia indica a presença de uma cisma entre pensamento, emoção e comportamento; e a desordem dissociativa ou dissociação se refere a dois ou mais processos mentais não associados ou não integrados (Asher, Fekadu, \& Hanlon, 2018; Ribáry, Lajtai, Demetrovics, \& Maraz, 2017). Além disso, ambos têm no ambiente um elemento comum: na esquizofrenia, fatores do ambiente (interno e externo) provocam um efeito patogênico sobre o sistema nervoso do paciente afetado; nas desordens dissociativas, uma das características essenciais é a interrupção das funções que integram a percepção do ambiente. O trauma, ocorrido na primeira infância, é um dos fatores ambientais de risco para esquizofrenia e condição para início de experiências dissociativas, como o TDI (Gibson, Alloy, \& Ellman, 2016; Negro Júnior, Negro, \&
Louzã, 1999; Van der Hart, 2012). Esses fatores integram o foco deste trabalho.

Outras comparações ora aproximam, ora distanciam o diagnóstico da esquizofrenia do diagnóstico da TDI: na esquizofrenia, falta consenso científico no tocante a suas bases etiológicas; no TDI, profissionais da saúde mental defendem que ele pode ser causado por "praticantes", e outros são céticos quanto à existência de tais transtornos. $\mathrm{Na}$ esquizofrenia, o diagnóstico geralmente leva algum tempo porque, na maioria dos casos, pessoas com esse transtorno não têm consciência de que suas dificuldades são causadas por uma doença que exige atenção médica e não procuram um especialista; no TDI, o diagnóstico só ocorre, em média, seis a oito anos após o surgimento dos sintomas. Na esquizofrenia, a precisão diagnóstica muitas vezes ainda revela incipiência científica justamente por sua complexidade; no TDI, pode-se dizer, relativamente, que ocorre o mesmo, devido ao negativismo que o envolve, quanto a crenças de que suas causas são iatrogênicas (Cordeiro, Silva, \& Vallada, 2012; Lewis et al., 1997; Yu et al., 2010). Ambas as patologias são cercadas de incertezas, apesar do reconhecido avanço da tríade ciência-tecnologia-fenomenologia.

Tanto a esquizofrenia quanto o TDI têm relatos de traumas em seu histórico. Na primeira, citam-se como fatores traumáticos a negligência emocional, o abandono, o desamparo e a rejeição na infância, associados ao surgimento de sintomas psicóticos ou repercussões nocivas na fase adulta. A extensão dos danos decorrentes desses fatores varia de acordo com a vulnerabilidade de cada paciente, uma vez que os traumas podem se referir a um único acontecimento externo, como abuso sexual ou estupro ou a um acúmulo deles (Gibson, Alloy, \& Ellman, 2016; Kumar, Castellani, Maiti, O'reilly, \& Singh, 2013).

O TDI resulta de um desequilíbrio que afeta a personalidade, como os traumas. Indivíduos com esse transtorno relatam, com muita frequência, experiências de severo abuso físico e sexual, especialmente na infância. Inclusive, em sua forma severa, esse transtorno é um mecanismo de sobrevivência psicológica, uma defesa mobilizada pelo sujeito desprotegido para resistir à invasão da dor de traumas reais. A personalidade emocional da identidade ficou aprisionada no período do trauma e revive de forma crônica as experiências dolorosas, engajada em defesas individuais como luta, fuga ou colapso. A personalidade original se fragmenta, tentando manter o próprio funcionamento, enquanto evita memórias traumáticas (Moskowitz, 2011; Van der Hart, 2012).

Tanto em um caso quanto no outro, trata-se de um diagnóstico difícil e complexo. Na esquizofrenia, ele continua controverso, diante de argumentos contrários de que não há evidências empíricas para seu suporte (o diagnóstico). Na ausência de marcadores neurobiológicos consistentes, o diagnóstico depende de apreciação subjetiva quanto aos sinais e sintomas apontados no DSM-V e 
na Classificação Internacional de Doenças (CID-10). A identificação de uma fisiopatologia, associada a parâmetros biológicos inequívocos continua sendo um desafio (Schmitt, Reich-Erkelenz, Gebick-Härter, \& Falkai, 2012). No TDI, deve haver outros cuidados por parte do médico no diagnóstico, no sentido de desencorajar o pensamento estereotipado sobre esse transtorno (causas iatrogênicas, por exemplo). O diagnóstico exige conhecimento de questões específicas da dissociação (Dorahy et al., 2014; Kluft, 1991).

A questão desta pesquisa centra-se no diagnóstico dessas patologias que, se equivocado, faz com que pacientes com TDI sejam diagnosticados com esquizofrenia. Um diagnóstico é constituído de ideias fundamentais para o desenvolvimento do trabalho médico, as quais são baseadas em fenômenos que ocorrem com a pessoa e que são agrupados e classificados conforme especificidades. Esses agrupamentos se dão por traços ou sinais que guardam alguma semelhança entre si e, por essa razão, fazem com que patologias possam ser confundidas em seu diagnóstico, como ocorre com a esquizofrenia e com o TDI. Nesse último caso, há uma noção de "janela de diagnosticabilidades", devido a seus sintomas encobertos e à dissimulação dos pacientes para encobri-lo, além da suspeita de apresentação polissintomática (Dorahy et al., 2014; Leonard \& Tiller, 2016).

Ocorre que a esquizofrenia e o TDI partilham alguns sintomas, apesar da divergência de visão entre os autores que leva à discussão do assunto: o TDI tem componentes semelhantes aos encontrados na esquizofrenia, no transtorno afetivo bipolar, no transtorno de personalidade borderline e em outras síndromes. Porém, incorpora características próprias, como alterações próprias de voz e do padrão da escrita, e amnésias (Lewis et al., 1997). Além de ouvir vozes (como os esquizofrênicos), pacientes com TDI podem ter alucinações visuais, táteis, olfativas e gustativas. Mas esses sintomas alucinatórios diferem das alucinações típicas de perturbações psicóticas como as da esquizofrenia. Porém, vivências alucinatórias não necessariamente implicam um diagnóstico patológico de esquizofrenia.

Eventos traumáticos ocorridos no passado podem estar vinculados ao desenvolvimento da esquizofrenia, e a gravidade, a duração e a proximidade da exposição de um indivíduo a um evento traumático são fatores determinantes para a probabilidade de surgimento da dissociação patológica (Dell, 2002; Gibson, Alloy, \& Ellman, 2016). Em termos de violência externa, o abuso infantil, físico ou psíquico pode ser fator etiológico determinante do desencadeamento do TDI, relacionado com a dissociação patológica da consciência, que também pode estar associada à base etiológica da esquizofrenia (Haddock, 2001; Putnam, 1989; Rich, 2005).

Na perspectiva de um diagnóstico diferencial entre esses transtornos, a Técnica de Rorschach pode ser utilizada. Trata-se de um instrumento golden standard da Psicologia, um dos testes psicológicos mais utilizados mundialmente para fins psicodiagnósticos, que também avalia déficits neurocognitivos e esclarece componentes profundos do comportamento humano, principalmente quando o sujeito avaliado não tem consciência daquilo que está sendo medido. Apesar de sua margem de limitação (como em qualquer instrumento de avaliação do comportamento humano e dos processos cognitivos em geral), essa técnica fornece subsídios qualitativos e quantitativos para avaliar a dinâmica da personalidade e seu funcionamento psicodinâmico (Mihura, Bombel, Dumitrascu, Roy, \& Meadows, 2018).

Considerando as informações prévias, entre as informações qualitativas estão os chamados fenômenos especiais, representados por condutas e verbalizações do indivíduo, não classificáveis pelos signos utilizados habitualmente. Atualmente, esses fenômenos, também chamados de "fatores imponderáveis", compõem uma lista de quase uma centena e meia de exemplos, embora ainda haja discordância quanto a sua validade. Rorschach considera que a identificação de fenômenos especiais em pacientes é fundamental para o diagnóstico por meio dessa técnica, mesmo que pesquisadores discordem quanto a sua inserção no protocolo (Gacono, Loving, \& Bodholdt, 2001; Mihura, Bombel, Dumitrascu, Roy, \& Meadows, 2018).

Por meio dos fatores especiais ou imponderáveis, são avaliadas as respostas dadas pelo paciente na interpretação qualitativa das lâminas ou pranchas ou cartões apresentados a ele. Tais lâminas constituem um instrumento auxiliar de diagnóstico da técnica, e as imagens contidas nelas são manchas sem significado predeterminado; logo, todas as respostas são produto da percepção interpretativa dos pacientes. Quando apresentadas ao paciente e se pergunta a ele "o que você vê" (nas lâminas), a demanda é de que ele recorte e construa um espaço e o articule em uma superfície limitada. O modo como ele recorta indica o que ele vê, já que não é sujeito passivo. O olhar ordena o visível e organiza a experiência, isto é, o sujeito contemplador, por meio de seus códigos invisíveis, ordena o visível. As lâminas (pranchas ou cartões) constituem um espaço de emersão de matéria-prima, sem codificação prévia. Sua interpretação é um esforço de resposta, tarefa de caráter mais amplo e complexo de dar sentido ao mundo exterior e a sua construção material e de vínculos. Esse processo nem sempre se dá sem dificuldade, no que diz respeito às particularidades da percepção (Gacono, Loving, \& Bodholdt, 2001).

Para se referir à combinação deformante e ilógica de duas ou mais ideias obtidas com a interpretação das figuras das lâminas, Rorschach usou o termo "contaminação", que corresponde, de certa forma, à condensação com que Freud define um dos processos de funcionamento mental que envolve tanto expressões patológicas como adaptativas (inclusive criativas, como o chiste e a metáfora). A resposta contaminada envolve fluidez entre os limites das ideias ou conceitos, de modo que dois ou mais objetos, abstraídos de uma área da mancha das lâminas, fundem-se entre si para constituir uma só ideia ou percepção, independente de haver 
qualquer lógica convencional nessa junção. Isso representa o distúrbio mais patológico do pensamento, porque representa um déficit da capacidade de suprimir impressões incompatíveis ou conflitantes, segundo Rorschach. A contaminação pode ser principalmente verbal (decorrente de neologismos, como na referência a órgãos do corpo: fígado, pulmões e "intestículos", condensando intestinos e testículos), perceptual (fusão de imagens diferentes, como morcego com olhos brancos) ou conceitual (como conceitos incompatíveis como um morcego morto estava voando), mas também pode combinar alguns desses aspectos (Vila \& Lunazzi, 2010).

Neste estudo clínico, objetivou-se investigar, com base na Técnica de Rorschach e nos elementos anamnésicos, a prevalência do TDI em pacientes diagnosticados com esquizofrenia.

\section{MÉTODO}

O método adotado foi série de casos, que requer um número mínimo de dez pacientes para a sistematização. Trata-se de um estudo descritivo, não-controlado. A abordagem foi qualitativa/quantitativa, sendo observados sintomas psicológicos e psiquiátricos envolvidos na esquizofrenia e no TDI, método de Rorschach, interpretação dos resultados conforme critérios das patologias enfocadas, principalmente o DSM-5 e CID 10.

Participaram do estudo dez pessoas com o diagnóstico de esquizofrenia (CID 10, F.20), feito por psiquiatras que acompanharam os pacientes ao longo da vida e corroborados pelos especialistas da instituição, com características paranoides, sendo cinco homens e cinco mulheres. Todos tinham acompanhamento psiquiátrico regular, faziam uso de medicação contínua para tratamento da patologia, não apresentavam problemas de visão e tinham como comorbidade o fator ansiedade.

Os participantes foram selecionados de forma aleatória pelo pesquisador. Seus nomes foram apresentados pela coordenadora do Centro de Convivência e Atenção Psicossocial (Mansão Vida) (DF) onde eles se encontravam internados em núcleos. Essas pessoas atenderam aos seguintes critérios de inclusão: registro em prontuário de internação na Instituição; maioridade (21 anos); diagnóstico de esquizofrenia há, pelo menos, cinco anos; concordância sobre a participação no estudo. Escolheu-se a modalidade paranoide nos pacientes esquizofrênicos, com a presença de três fatores que os colocavam em sintonia com o contexto do TDI: (1) ideias delirantes relativamente estáveis; (2) alucinações, particularmente auditivas; (3) perturbação das percepções.

Os dados foram coletados no setting desse Centro, e os instrumentos de coleta utilizados foram: entrevista clínica e Técnica de Rorschach, na abordagem de B. Klopfer, validada por Vaz (1997), além do atlas de Augras (2004). As entrevistas foram individuais, semiestruturadas em duas partes: a primeira voltada para uma identificação do paciente, motivo da internação, histórico atual, histórico pessoal e familiar (Carretoni Filho \& Prebianchi, 1999); a segunda orientada para possíveis contextos dissociativos experienciados. Para tal, foram inseridos elementos selecionados da Escala de Experiências Dissociativas (DES) de Putnam (1992), adaptados ao tipo de pesquisa. A entrevista foi um importante meio utilizado para conhecimento do sujeito, por se tratar de um instrumento importante de conhecimento interpessoal, facilitando a apreensão de elementos que poderiam contribuir para a identificação do fenômeno pesquisado, além de auxiliar a construção do perfil do entrevistado.

Os sujeitos foram avaliados individualmente, em tempo livre, e a duração estimada foi de quatro horas por paciente. O tempo para conclusão da tarefa variou para mais ou para menos, conforme as condições do sujeito avaliado. A sequência do processo de avaliação dos participantes foi feita na seguinte ordem: (1) assinatura do Termo de Consentimento Livre e Esclarecido (TCLE) para participação na pesquisa, pelo paciente e/ou resposável legal; (2) anamnese e entrevista clínica semiestruturada; (3) aplicação do teste de Rorschach, abordagem de B. Klopfer. Todos os protocolos foram codificados pelo pesquisador. Uma psicóloga, treinada na correção do Método, Sistema Klopfer, analisou os citados protocolos para que a análise fosse realizada da forma mais fidedigna. Não houve discordância entre os profissionais, o que afastou a necessidade de um terceiro avaliador.

A proposta desta pesquisa foi submetida ao Conselho de Ética em Pesquisa da Universidade de Brasília, Faculdade de Ciências da Saúde. O estudo recebeu voto favorável a sua realização, conforme Parecer $n^{\circ} 421.122$. Princípios éticos relativos à pesquisa envolvendo seres humanos, inclusive aqueles que dizem respeito ao sigilo dos pacientes, foram obedecidos em todos os momentos.

\section{RESULTADOS}

\section{Dados da Anamnese}

Para a finalidade desta pesquisa, cada integrante da amostra foi identificado da seguinte forma: Paciente 1;
Paciente 2; Paciente 3; Paciente 4; Paciente 5; Paciente 6; Paciente 7; Paciente 8; Paciente 9; Paciente 10. A tabela 1 apresenta, em dois grupos - (A) Elementos Não Dissociativos e (B) Elementos Dissociativos -, os 
Tabela 1

Elementos da Anamnese - Aspectos Relevantes.

\begin{tabular}{|c|c|c|c|c|c|c|c|c|c|c|}
\hline \multirow{2}{*}{$\begin{array}{l}\text { Grupo “A” } \\
\text { Indicadores Não Dissociativos }\end{array}$} & \multicolumn{10}{|c|}{ Pacientes } \\
\hline & 1 & 2 & 3 & 4 & 5 & 6 & 7 & 8 & 9 & 10 \\
\hline 1- Alucinações (auditivas, visuais ou olfativas). & $\mathrm{X}$ & $\mathrm{X}$ & $\mathrm{X}$ & $\mathrm{X}$ & $\mathrm{X}$ & $\mathrm{X}$ & $\mathrm{X}$ & $\mathrm{X}$ & $\mathrm{X}$ & $\mathrm{X}$ \\
\hline 2- Baixo rendimento escolar ao longo dos anos. & $\mathrm{X}$ & $\mathrm{X}$ & $\mathrm{X}$ & & & $\mathrm{X}$ & $\mathrm{X}$ & $\mathrm{X}$ & $\mathrm{X}$ & \\
\hline 3- Curso ou conteúdo do pensamento prejudicados. & $\mathrm{X}$ & $\mathrm{X}$ & $\mathrm{X}$ & $\mathrm{X}$ & $\mathrm{X}$ & $\mathrm{X}$ & $\mathrm{X}$ & $\mathrm{X}$ & $\mathrm{X}$ & $\mathrm{X}$ \\
\hline $\begin{array}{l}\text { 4- Delírios de: controle, grandeza, perseguição, } \\
\text { culpa, pecado ou somático. }\end{array}$ & $\mathrm{X}$ & $\mathrm{X}$ & $\mathrm{X}$ & $\mathrm{X}$ & $\mathrm{X}$ & $\mathrm{X}$ & $\mathrm{X}$ & $\mathrm{X}$ & $\mathrm{X}$ & $\mathrm{X}$ \\
\hline $\begin{array}{l}\text { 5- Dificuldades interpessoais com as figuras } \\
\text { parentais e/ou com membros do núcleo familiar. }\end{array}$ & $\mathrm{X}$ & $\mathrm{X}$ & $\mathrm{X}$ & $\mathrm{X}$ & $\mathrm{X}$ & $\mathrm{X}$ & $\mathrm{X}$ & $\mathrm{X}$ & $\mathrm{X}$ & $\mathrm{X}$ \\
\hline 6- Esquiva de contatos sociais. & $\mathrm{X}$ & $\mathrm{X}$ & $\mathrm{X}$ & $\mathrm{X}$ & $\mathrm{X}$ & $\mathrm{X}$ & $\mathrm{X}$ & $\mathrm{X}$ & $\mathrm{X}$ & $\mathrm{X}$ \\
\hline $\begin{array}{l}\text { 7- Falta de controle das emoções e dos impulsos na } \\
\text { maioria das ocasiões adversas. }\end{array}$ & $\mathrm{X}$ & $\mathrm{X}$ & $\mathrm{X}$ & $\mathrm{X}$ & $\mathrm{X}$ & $\mathrm{X}$ & $\mathrm{X}$ & $\mathrm{X}$ & $\mathrm{X}$ & $\mathrm{X}$ \\
\hline $\begin{array}{l}\text { 8- Ideação suicida c/possibilidade de conversão em } \\
\text { ato. }\end{array}$ & $\mathrm{X}$ & $\mathrm{X}$ & $\mathrm{X}$ & $\mathrm{X}$ & $\mathrm{X}$ & $\mathrm{X}$ & $\mathrm{X}$ & $\mathrm{X}$ & $\mathrm{X}$ & $\mathrm{X}$ \\
\hline 9- Núcleo familiar disfuncional. & $\mathrm{X}$ & $\mathrm{X}$ & $\mathrm{X}$ & $\mathrm{X}$ & $\mathrm{X}$ & $\mathrm{X}$ & $\mathrm{X}$ & $\mathrm{X}$ & $\mathrm{X}$ & $\mathrm{X}$ \\
\hline 10- Escuta de vozes $(*)$ & $\mathrm{X}$ & $\mathrm{X}$ & $\mathrm{X}$ & $\mathrm{X}$ & $\mathrm{X}$ & $\mathrm{X}$ & $\mathrm{X}$ & $\mathrm{X}$ & $\mathrm{X}$ & $\mathrm{X}$ \\
\hline 11- Relações afetivas instáveis. & $\mathrm{X}$ & $\mathrm{X}$ & $\mathrm{X}$ & $\mathrm{X}$ & $\mathrm{X}$ & $\mathrm{X}$ & $\mathrm{X}$ & $\mathrm{X}$ & $\mathrm{X}$ & $\mathrm{X}$ \\
\hline $\begin{array}{l}\text { 12- Uso de SPA's, lícitas e/ou ilícitas em alguma } \\
\text { etapa da vida. }\end{array}$ & $\mathrm{X}$ & $\mathrm{X}$ & $\mathrm{X}$ & & & $\mathrm{X}$ & $\mathrm{X}$ & $\mathrm{X}$ & $\mathrm{X}$ & $\mathrm{X}$ \\
\hline Quantidade de indicadores do Grupo 'A': & 12 & 12 & 12 & 10 & 10 & 12 & 12 & 12 & 12 & 11 \\
\hline Percentuais obtidos no Grupo 'A': & $100 \%$ & $100 \%$ & $100 \%$ & $83 \%$ & $83 \%$ & $100 \%$ & $100 \%$ & $100 \%$ & $100 \%$ & $91 \%$ \\
\hline \multirow{2}{*}{$\begin{array}{l}\text { Grupo "B" } \\
\text { Indicadores Dissociativos }\end{array}$} & \multicolumn{10}{|c|}{ Pacientes } \\
\hline & 1 & 2 & 3 & 4 & 5 & 6 & 7 & 8 & 9 & 10 \\
\hline $\begin{array}{l}\text { 1- Às vezes, sentimento de que seu corpo não the } \\
\text { pertence. }\end{array}$ & $\mathrm{X}$ & & & $\mathrm{X}$ & $\mathrm{X}$ & $\mathrm{X}$ & $\mathrm{X}$ & $\mathrm{X}$ & & \\
\hline $\begin{array}{l}\text { 2- Surpresa diante de roupas que não se lembra } \\
\text { ter vestido. }\end{array}$ & & & & & $\mathrm{X}$ & & & & & \\
\hline 3- É comum não se reconhecer ao espelho. & & & $\mathrm{X}$ & $\mathrm{X}$ & $\mathrm{X}$ & & & & & \\
\hline $\begin{array}{l}\text { 4- Em algumas ocasiões, sentimento de que se está } \\
\text { fora do corpo, observando-se como se fosse outra } \\
\text { pessoa ou sentindo que as pessoas, as coisas e o } \\
\text { mundo ao redor não são reais. }\end{array}$ & & $\mathrm{X}$ & & $\mathrm{X}$ & $\mathrm{X}$ & & & & $\mathrm{X}$ & $\mathrm{X}$ \\
\hline 5- Já se viu num lugar e não sabe como chegou lá. & & & & & $\mathrm{X}$ & & & & & \\
\hline $\begin{array}{l}\text { 6- Muitas vezes não percepção da passagem do } \\
\text { tempo ou falta compreensão por que o tempo } \\
\text { transcorre tão rapidamente. }\end{array}$ & & & & $\mathrm{X}$ & $\mathrm{X}$ & & & & & $\mathrm{X}$ \\
\hline $\begin{array}{l}\text { 7- Percepção de objetos novos entre seus pertences } \\
\text { e não se recorda de tê-los comprado. }\end{array}$ & & & & & $\mathrm{X}$ & & & & & \\
\hline $\begin{array}{l}\text { 8- Pessoas desconhecidas chamam você por outro } \\
\text { nome ou dizem que já o encontrou antes. }\end{array}$ & & & & $\mathrm{X}$ & $\mathrm{X}$ & & & & & \\
\hline Quantidade de indicadores do Grupo 'B': & 01 & 01 & 01 & 05 & 08 & 01 & 01 & 01 & 01 & 02 \\
\hline Percentuais obtidos no Grupo 'B': & $12 \%$ & $12 \%$ & $12 \%$ & $62 \%$ & $100 \%$ & $12 \%$ & $12 \%$ & $12 \%$ & $12 \%$ & $25 \%$ \\
\hline Total de indicadores dos grupos $A+B$ & 13 & 13 & 13 & 15 & 18 & 13 & 13 & 13 & 13 & 13 \\
\hline \% geral dos indicadores observados ' $A$ '+'B': & $65 \%$ & $65 \%$ & $65 \%$ & $75 \%$ & $90 \%$ & $65 \%$ & $65 \%$ & $65 \%$ & $65 \%$ & $65 \%$ \\
\hline
\end{tabular}

Fonte: Elaborado pelo pesquisador

Nota. Os elementos do Grupo "B" foram adaptados de Putnam (1992).

(*) Todos os pacientes, exceto o de número 5 , disseram que as vozes ouvidas são provenientes de fora da cabeça. $O$ paciente 5 disse que as vozes ouvidas são oriundas de dentro da sua cabeça. 
aspectos relevantes identificados nas entrevistas clínicas semiestruturadas individuais com pacientes esquizofrênicos, bem como suas totalizações e percentuais específicos.

Pelos resultados observados na tabela, todos os pacientes apresentaram significativa similaridade de resultados em relação aos fatores do Grupo "A", classificados como elementos anamnésicos não dissociativos. Ou seja: o conjunto de pacientes obteve percentuais equivalentes $(\geq$ $80 \%$ ) nesse primeiro agrupamento, fato que incluiu todas as características diagnósticas da esquizofrenia e do TDI, cujos quadros clínicos apresentaram indicadores semelhantes. Não houve a mesma semelhança nos resultados em relação aos pacientes do Grupo "B", categorizados como elementos anamnésicos dissociativos. Nesse segundo agrupamento, houve discrepância em relação aos resultados dos pacientes 4 e 5, que demonstraram sintomas dissociativos mais acentuados do que os outros, principalmente o 5 .

Em relação ao total geral de indicadores e aos respectivos percentuais, também o paciente 5 apresentou a maior discrepância do conjunto de fatores observados, principalmente no que se referiu aos indicadores do Grupo "B". O paciente 4 demonstrou dessemelhança de características em relação aos três primeiros (pacientes 1, 2 e 3) e aos cinco últimos (pacientes 6, 7, 8, 9 e 10). Porém, suas diferenças foram discretas. Tais resultados, sugestivos da diversidade de sinais e sintomas que envolveram os dois tipos de transtorno abordados nesta pesquisa, realçaram a necessidade de investigações mais aprofundadas em relação aos pacientes em foco, para fins de diagnóstico diferencial.

\section{Indicadores da Esquizofrenia e do TDI no Rorschach e Critérios Diagnósticos}

Com base no Método de Rorschach, apresentam-se na tabela 2, os mesmos sinais típicos que caracterizam a síndrome esquizofrênica e o TDI. No TDI, a correlação de tais sinais com esse transtorno é fruto de observações da prática clínica do pesquisador e que não há, até o momento, comprovações científicas nesse sentido no cenário nacional. Em seguida, também de acordo com a experiência clínica do pesquisador relacionada com o TDI, são descritos os critérios utilizados no âmbito desta pesquisa, para fins de diagnóstico diferencial dos transtornos investigados.

Em conformidade com o apresentado na tabela 3, os sete primeiros indicadores, principais ou indispensáveis, obrigatoriamente devem estar presentes nos psicogramas de Rorschach de quaisquer pacientes esquizofrênicos (Vaz, 1997). Também foram encontrados em pacientes múltiplos, objeto de tratamento na prática clínica do pesquisador. Os demais, denominados indicadores secundários, também precisaram ser identificados nos protocolos desses pacientes, porém não de forma tão rigorosa em termos quantitativos.

Para que um paciente fosse diagnosticado portador do transtorno psicótico da esquizofrenia, além de $100 \%$ dos indicadores obrigatórios (principais ou indispensáveis)
Tabela 2

Sinais Típicos da Esquizofrenia e do TDI no Rorschach.

Indicadores principais (obrigatórios ou indispensáveis)

1- Baixo número de respostas de boa qualidade.

2- Elevado número de respostas confabulatórias e/ou contaminadas.

3- Ideias de referência ou de autorreferência.

4- Número de respostas populares muito baixos.

5- Presença de respostas abstratas e/ou absurdas.

6- \% de F- elevado (percentagem alta de formas mal vistas).

7- \% de F+ baixo ou inexistente

(percentagem baixa de formas bem vistas).

Indicadores secundários (não obrigatórios)

1- Aumento das respostas G (+ de 30\%) com diminuição de sua qualidade.

2- Críticas ao examinador.

3- Escassas interpretações de movimento humano (M).

4- Olhada no verso do cartão, podendo demonstrar comportamento enfadonho (monotonia).

5- Olhada no que está sendo escrito pelo examinador.

6- Perseveração de conteúdo durante várias pranchas seguidas, às vezes em todas as pranchas.

7- Presença de conteúdos anatômicos e de animal, que também podem estar associados a respostas de posição (PO).

8- Presença de Cor Nomeada.

9- Presença de neologismos, assim como nas respostas com conteúdo simbólico (muitas vezes sexuais e bizarros)

10- Presença de respostas anatômicas (ou de transposição) pouco usuais, estranhas e absurdas.

11- Presença de respostas de posição (PO).

12- Presença de respostas que fazem referência à cor nas pranchas escuras (Ex: "Sangue" numa prancha preta ou borboleta vermelha na prancha $\mathrm{V}$. Tais respostas não são classificáveis nas de cor e sim nas $C^{\prime}$ ).

13- Somatório de $\mathrm{C}+\mathrm{CF}>\mathrm{FC}$ (ausência da combinação forma e cor (FC) ou, de outro modo, as respostas de forma-cor FC encontram-se diminuídas em relação as CF (cor-forma) e as C (cor pura).

14- Sucessão desagregada (cortada) ou assistemática.

15- Tipo de vivência, ou Tipo de Ressonância Íntima (TRI), coartado / coartativo.

Fonte: Elaborada pelo pesquisador

citados, estimou-se, neste trabalho, um percentual aproximado de $60 \%$ dos sinais típicos secundários para se estabelecer o diagnóstico diferencial dos quadros clínicos enfocados.

Essa estimativa baseou-se nos seguintes critérios: primeiro, em resultados de estudos referenciados por Vaz (1997), os quais constataram a presença de sinais típicos da esquizofrenia, ora semelhantes, ora diferentes, em pacientes submetidos ao Rorschach, sem distinção entre principais e secundários. Pelas referências de Vaz, Del Roncal (1940) encontrou doze sinais; Piotrowsky (1945), três; Bochner 
e Halpner (1945), três, e Klopfer et al (1956), um. Desse total de 19 sinais, 13 ou $59,1 \%$ se encontram na tabela 3 , entre os 22 observados pelo pesquisador em sua prática clínica, distribuídos também entre principais e secundários (respectivamente: 2, 3, 5 e 7; 1, 4, 6, 8, 10, 11, 13 e 15). Segundo, tomou-se como referência $60 \%(59,1 \%$ dos primários e secundários constantes da tabela 3 arredondados) para aplicar apenas aos sinais secundários, como forma de prover os resultados da pesquisa empírica de maior margem de segurança. Considerou-se, então, que um mínimo de $60 \%$ de sinais típicos secundários deveria ser encontrado nos referidos psicogramas, para se fechar o diagnóstico da esquizofrenia e do TDI.

Desta forma, para fins de diagnóstico diferencial nesta pesquisa, foram considerados os seguintes critérios para um paciente ser enquadrado no espectro da esquizofrenia, com base na Técnica de Rorschach e na entrevista clínica semiestruturada:

1. percentual $\geq 80 \%$ dos indicadores não dissociativos da Tabela 1;

2. percentual $\geq 10 \% \mathrm{e} \leq 50 \%$ do total de indicadores dissociativos da tabela 1 ;

3. percentual $\leq 70 \%$ do total geral de indicadores da tabela 1;
4. $100 \%$ dos sinais típicos obrigatórios (principais ou indispensáveis) da tabela 2 ;

5. percentual $\geq 60 \%$ dos sinais típicos secundários da tabela 2 (9 indicadores ou mais);

6. percentual $\geq 72 \%$ ( 16 indicadores ou mais) do total (22) de sinais especificados da tabela 2.

$\mathrm{Na}$ experiência clínica do pesquisador, os critérios do Rorschach relativos ao TDI e à esquizofrenia são semelhantes, o que requer maior atenção dos profissionais, no sentido de evitar falhas diagnósticas. No entanto, um pormenor de fundamental importância pode corroborar o diagnóstico diferencial entre essas duas síndromes: o fator dissociativo, destacado nos retângulos das listas de enumeração dos critérios diagnósticos.

Neste estudo, para um paciente passar do espectro da esquizofrenia para o do TDI com uma margem de segurança significativa para fins de diagnóstico diferencial, atestou-se a existência dos seguintes critérios:

1. percentual $\geq 80 \%$ dos indicadores não dissociativos da tabela 1 ;

2. percentual $\geq 85 \%$ do total de indicadores dissociativos da tabela 1 ;

3. percentual $\geq 85 \%$ do total geral de indicadores da tabela 1;

Tabela 3

Resultados Condensados

\begin{tabular}{|c|c|c|c|c|c|c|}
\hline \multirow[b]{2}{*}{ Pacientes } & \multicolumn{3}{|c|}{ Retirados da Tabela 1} & \multicolumn{3}{|c|}{ Retirados do modelo da Tabela 2 correspondente } \\
\hline & $\begin{array}{c}\text { Indicadores Não } \\
\text { Dissociativos } \\
\text { (A) }\end{array}$ & $\begin{array}{c}\text { Indicadores } \\
\text { Dissociativos } \\
\text { (B) } \\
\end{array}$ & $\mathbf{A}+\mathbf{B}$ & $\begin{array}{l}\text { Indicadores } \\
\text { Principais } \\
\text { (C) }\end{array}$ & $\begin{array}{c}\text { Indicadores } \\
\text { Secundários } \\
\text { (D) }\end{array}$ & $\mathbf{C}+\mathbf{D}$ \\
\hline 1 & $100 \%$ & $12 \%$ & $65 \%$ & $100 \%$ & $86 \%$ & $90 \%$ \\
\hline 2 & $100 \%$ & $12 \%$ & $65 \%$ & $100 \%$ & $73 \%$ & $81 \%$ \\
\hline 3 & $100 \%$ & $12 \%$ & $65 \%$ & $100 \%$ & $66 \%$ & $77 \%$ \\
\hline 4 & $83 \%$ & $62 \%$ & $80 \%$ & $100 \%$ & $53 \%$ & $68 \%$ \\
\hline 5 & $83 \%$ & $100 \%$ & $90 \%$ & $100 \%$ & $66 \%$ & $77 \%$ \\
\hline 6 & $100 \%$ & $12 \%$ & $65 \%$ & $100 \%$ & $80 \%$ & $86 \%$ \\
\hline 7 & $100 \%$ & $12 \%$ & $65 \%$ & $100 \%$ & $80 \%$ & $86 \%$ \\
\hline 8 & $100 \%$ & $12 \%$ & $65 \%$ & $100 \%$ & $73 \%$ & $81 \%$ \\
\hline 9 & $100 \%$ & $12 \%$ & $65 \%$ & $100 \%$ & $80 \%$ & $86 \%$ \\
\hline 10 & $91 \%$ & $25 \%$ & $65 \%$ & $100 \%$ & $86 \%$ & $90 \%$ \\
\hline Critérios & $\begin{array}{l}\geq 80 \% \\
\text { ( } \geq 10 \text { indicadores) } \\
=\text { Esquizofrênico } \\
\text { e Múltiplo }\end{array}$ & $\begin{array}{l}\geq 10 \% \text { e } \leq 50 \% \\
\text { (de } 1 \text { a } 4 \\
\text { indicadores) } \\
=\text { Esquizofrênico } \\
\geq 51 \% \text { e } \leq 84 \% \\
\text { (de } 5 \text { a } 6 \\
\text { indicadores) } \\
=\text { Outro diagnóstico } \\
\geq 85 \% \\
\text { ( } \geq 7 \text { indicadores) } \\
=\text { Múltiplo }\end{array}$ & $\begin{array}{l}\text { Até } 70 \% \\
\text { (Até } 14 \text { indicadores) } \\
=\text { Esquizofrênico } \\
\geq 71 \% \text { e } \leq 84 \% \\
\text { (de } 15 \text { a } 16 \\
\text { indicadores) } \\
=\text { Outro diagnóstico } \\
\geq 85 \% \\
\text { ( } \geq 17 \text { indicadores) } \\
=\text { Múltiplo }\end{array}$ & $\begin{array}{l}100 \% \\
\text { (todos os } 7 \\
\text { indicadores } \\
\text { indispensáveis) } \\
=\text { Esquizofrênico } \\
\text { e Múltiplo }\end{array}$ & $\begin{array}{l}\geq 60 \% \\
\text { ( } \geq 9 \text { indicadores) } \\
=\text { Esquizofrênico e } \\
\text { Múltiplo }\end{array}$ & $\begin{array}{l}\geq 72 \% \\
(\geq 16 \text { indicadores) } \\
=\text { Esquizofrênico e } \\
\text { Múltiplo }\end{array}$ \\
\hline
\end{tabular}

Fonte: Elaborada pelo pesquisador 
4. ocorrência de fenômenos dissociativos durante a aplicação do Método de Rorschach;

5. $100 \%$ dos sinais típicos obrigatórios (principais) da tabela 2;

6. percentual $\geq 60 \%$ dos sinais típicos secundários da tabela 2 ( 9 indicadores ou mais);

7. percentual $\geq 72 \%$ ( 16 indicadores ou mais) do total (22) de sinais especificados da tabela 2.

Logicamente, não sendo possível o enquadramento de um paciente em nenhum dos dois quadros clínicos mencionados, sugere-se que ele seja portador de um transtorno com outra classificação nosológica.

A tabela 3 engloba os resultados condensados obtidos dos pacientes pesquisados, em relação à presença de indicadores dissociativos, principais e secundários, bem como os sinais típicos da esquizofrenia e do TDI no Rorschach, tendo como parâmetro os percentuais adotados para a pesquisa. Em seguida, fez-se uma análise dos resultados para verificar a existência de falhas diagnósticas ou para confirmar se os diagnósticos diferenciais, previamente estabelecidos para cada um dos pacientes esquizofrênicos, estão em consonância com os critérios adotados nesta pesquisa.

De acordo com esses dados, verificou-se que os pacientes $1,2,3,6,7,8,9$ e 10 enquadraram-se no diagnóstico da esquizofrenia, segundo os critérios do Método de Rorschach utilizados no estudo. Já em relação aos pacientes 4 e 5, há discrepâncias diagnósticas. Os sintomas do paciente 4 não corresponderam aos da síndrome esquizofrênica, nem aos do TDI, sugerindo que seu transtorno pode se enquadrar em outra classificação nosológica, com características similares às dos transtornos aqui enfocados. Os resultados do paciente 5 permitiram dizer que, pelos sintomas, ele se encontra no espectro dissociativo, com características do TDI.

Em relação ao objetivo deste estudo, foram observadas duas diferenças em termos de diagnóstico diferencial, conforme os critérios adotados nesta pesquisa.

\section{DISCUSSÃO}

De forma sucessiva, discutem-se os dados obtidos na pesquisa de campo, com foco no objetivo proposto para o estudo, qual seja, investigar, com base no Método de Rorschach e em elementos anamnésicos, a prevalência do TDI em pacientes diagnosticados com esquizofrenia. Para tanto, primeiramente, são abordados os indicadores clínicos que caracterizam o diagnóstico diferencial de pacientes esquizofrênicos e de pacientes com TDI. Em seguida, são examinadas diferenças clínicas específicas que caracterizam tais transtornos, comparando os dados obtidos nas entrevistas e nos resultados do Rorschach com os aspectos teóricos encontrados na literatura nacional e na internacional destacada sobre os temas. Por fim, discute-se o enquadramento dos psicogramas de pacientes esquizofrênicos no domínio do TDI e averigua-se a ocorrência de eventuais falhas no diagnóstico de esquizofrenia dos pacientes pesquisados, seguindo a conclusão sobre a prevalência do TDI em pacientes diagnosticados com esquizofrenia.

Em termos dos indicadores clínicos examinados, apurouse a presença de idênticos parâmetros não-dissociativos entre os pacientes avaliados, diagnosticados com esquizofrenia. Como tais indicadores também integram o TDI, pela teoria levantada - mesmo sem haver pesquisas dessa natureza no âmbito nacional -, pode-se dizer que pacientes esquizofrênicos e pacientes com TDI têm alucinações, alterações no curso e no conteúdo do pensamento, delírios, dificuldades com as figuras parentais, esquiva social, emoções em desgoverno, ideação suicida, núcleo familiar disfuncional, escuta de vozes e labilidade afetiva. Segundo Kihlstrom, Glisky e Angiulo (1994), na esquizofrenia, as alucinações são precedidas de traumas geradores de perturbações sem controle, e no TDI, a dissociação é primeiro buscada na fantasia. Elis, Caponigro e Kring (2013) citam, ainda, na esquizofrenia: embotamento afetivo, pobreza de discurso, associabilidade - menor interesse, motivação ou vontade de estar com outras pessoas; anedonia, avolição.

No tocante aos fatores dissociativos investigados, foram verificados resultados discrepantes em termos quantitativos e qualitativos, demonstrando que pacientes esquizofrênicos apresentam características de dissociação patológica da consciência com grau significativamente menor que pacientes múltiplos. A diferença clínica mais proeminente observada entre as duas síndromes estudadas relaciona-se, exclusivamente, à dissociação patológica da consciência. Equivale a dizer que o aspecto dissociativo representa um dos fatores, se não o maior deles, que confunde os especialistas e provoca falhas diagnósticas facilmente.

Naturalmente, quando um paciente dissocia, suas realidades concreta e psíquica sofrem alterações. Em consequência, a atividade perceptiva, o funcionamento somático, as memórias, os hábitos e costumes, os conhecimentos ou uma combinação de tudo isso podem ser interpretados como alucinação ou delírio. No entanto, tais interpretações podem não ser indicadoras de esquizofrenia, como algumas vezes ocorre. Segundo Freud (1996) e Breuer, um mesmo indivíduo pode ter agrupamentos mentais relativamente independentes entre si, que aparecem espontânea e ocasionalmente; esses são descritos como exemplos de double consciense.

Várias explicações são encontradas na literatura para o fato de os pacientes dissociarem, e uma ou outra pode aplicar-se aos casos, de acordo com as circunstâncias. No caso de pacientes múltiplos, obrigatoriamente, há processos traumáticos no histórico de vida, sempre relacionados com a possibilidade de abuso sexual, rejeição, abandono ou desamparo a partir da infância. Há, então, no TDI, uma 
relação significativa entre trauma e dissociação, ou seja, entre processos traumáticos e tendências à perda de contato com a realidade. Isso significa dizer que não há possibilidade de uma pessoa desenvolver o TDI sem a presença de fatores traumáticos reais geradores de dissociações patológicas (Lewis et al., 1997; Putnam, 1989). As dissociações foram evidenciadas nos resultados do Rorschach, além de serem um fator basilar na observação do pesquisador, já que o objetivo do trabalho teve como um de seus escopos a dissociação, especificamente o TDI. Pacientes esquizofrênicos não necessariamente sofrem processos dissociativos durante a aplicação do método. Por outro lado, sem exceções conforme os resultados deste estudo -, pacientes múltiplos sempre dissociam durante a aplicação da técnica, em algum momento da aplicação.

Pode-se pensar que os estímulos eliciadores das pranchas do Rorschach desencadeiam processos inconscientes que acionam fatores traumáticos reais geradores de dissociações. Esses, por sua vez, fazem emergir alters, característica maior do TDI. A sequência dessa dinâmica ou encadeamento sucessivo - trauma real $\rightarrow$ dissociação patológica $\rightarrow$ emersão-imersão de personalidades alternativas - resulta na busca de um processo reverso (tratamento curativo) para $o$ transtorno, alcançado com a integração dos fragmentos do eu e, consequentemente, fusão das personalidades dissociadas.

No que concerne ao enquadramento dos psicogramas de pacientes esquizofrênicos no domínio do TDI ou a sua prevalência - número de casos de TDI entre pacientes esquizofrênicos -, constatou-se a convergência de apenas uma ocorrência nos resultados obtidos: o paciente 5. Esse caso denota, etiologicamente, fatores que devem estar presentes no indivíduo para ele desenvolver o TDI: ausência de carinho, calma e experiências restauradoras que não permitem à criança se recuperar de traumas (Kluf,1984); exposição da criança a um ambiente em que houve abuso imprevisível por parte dos pais, causando predisposição ou capacidade para dissociar em situações de estresse; graves eventos traumáticos indicam o primeiro surgimento de dissociação como mecanismo de fuga da dor ou enfrentamento do problema com outro alter (Braun, 1990); como violência externa, tem-se o abuso infantil, físico ou psíquico como fator etiológico de desencadeamento do TDI (Haddock, 2001; Rich, 2005). Trata-se de experiências muito graves que ocorrem na infância e ameaçam a estabilidade emocional, cognitiva e a segurança física do indivíduo. Pacientes com TDI manifestam sinais e sintomas como: experiências crônicas de equívocos de identidade e alterações no controle do corpo (Van der Hart, 2012).

Assim, observa-se que o diagnóstico menos preciso de um e de outro transtorno pode levar o curso da patologia a resultados bem distintos, conforme o tratamento adotado: na esquizofrenia, ele inclui a farmacologia, com a prescrição de antipsicóticos que inibem muito os receptores da dopamina das vias dopaminérgicas; neurolépticos, indicados para pacientes que não respondem aos antipsicóticos convencionais ou que sofrem efeitos colaterais indesejáveis. Também há indicação de psicoterapia, com a finalidade, entre outras, de interromper a perda da capacidade mental, preservando o contato com a realidade (Amato, Beasley, Hahn, \& Vernon, 2016; Kar, Barreto, \& Chandavarkar, 2016; Maramai et al., 2016).

No TDI, a forma de tratamento mais utilizada é a psicoterapia. A farmacologia é usada para auxiliar o controle dos sintomas da depressão e da impulsividade, não aliviando a dissociação. Antipsicóticos atípicos (ou de segunda geração) podem estabilizar o humor, moderar a ansiedade e sintomas intrusivos. Antagonistas opioides, como a naltrexona, mostram-se promissores no tratamento de diversos de seus sintomas, podendo reduzir índices de autoagressão (Brand, Loewenstein, \& Spiegel, 2014; Gentile \& Dillon, 2013; Lakshmanan, Meier, Meier, \& Lakshmanan, 2010).

Apesar de alguns sugerirem a possibilidade de terapia medicamentosa para as manifestações aqui apresentadas, percebe-se, entretanto, a pouca efetividade de um medicamento capaz de tratar o TDI de forma vantajosa e duradoura. Isso deve-se ao fato de ser grande o espectro sintomático do transtorno e há pouco conhecimento relativo à eletrofisiologia da desordem. Em algumas situações, o uso da medicação é ineficiente ou passível de risco, como por exemplo, quando não há suporte e apoio ante o contexto das múltiplas personalidades. Então, nesse caso, o uso pode ser feito à revelia do paciente, de acordo com a vontade de algumas personalidades alternativas (alters). Nem sempre uma determinada personalidade mostra-se colaborativa em relação à outra, podendo esconder remédios, alterar dosagens, provocar tragédias irreversíveis, entre outras (Zimerman, 1999).

Portanto, no tratamento psicológico desse transtorno, a abordagem mais comum considera os sintomas, visando assegurar não só a integridade do indivíduo, como reconectálo às múltiplas identidades, com o que ele tende a melhorar. Esse processo é conhecido como integração. A integração dos estados de identidade é a abordagem mais desejável do tratamento de TDI (Kluft, 2000).

Porém, mesmo sem ser especificamente objetivo de o trabalho verificar outros transtornos psicóticos, eventualmente confundidos com esquizofrenia em seu diagnóstico, não se pode deixar de registrar uma ocorrência nesse sentido: o paciente 4 apresentou resultados que não corresponderam aos da síndrome esquizofrênica, nem aos do TDI. Sims (1988) afirma que a fenomenologia da experiência ou aspectos transpessoais podem se assemelhar ou representar sintomas de outros problemas psiquiátricos. Assim, o resultado do paciente 4 sugere que seu transtorno mental pode ser enquadrado em outra classificação nosológica, com características similares às dos transtornos enfocados na testagem objeto deste trabalho.

Com isso, resume-se que o diagnóstico diferencial de dois pacientes apresenta falhas, e que o fato de uma 
dessas falhas não apontar o TDI (objetivo deste trabalho) não torna o resultado menos importante. Isso porque o diagnóstico é o ponto de partida para o tratamento e, consequentemente, a cura ou, ao menos, o controle da patologia. Nesse sentido, vale sempre alertar sobre a questão de que nem sempre experiências ditas anômalas são psicóticas, além de poderem ter causas variadas. Por isso, devem ser investigadas profundamente para evitar falhas diagnósticas. Como exemplo, citam-se as alucinações, que podem surgir em decorrência de diversos fatores, a saber: tumores, aneurismas, processos neurodegenerativos, processos tóxicos, distúrbios metabólicos, derrames, doenças infecciosas, traumas psicológicos, experiências religiosas ou transpessoais.

Embora este trabalho se volte de modo mais específico à área psiquiátrica, no que diz respeito ao diagnóstico da esquizofrenia, os efeitos desse diagnóstico, adequado ou não, são psicológicos e se reproduzem na vida pessoal, social e profissional do indivíduo. Cada área é autônoma, em termos psiquiátricos e psicológicos, porém, não devemos nos esquecer de que os efeitos de uma disciplina recaem sobre a outra. No atual estágio em que se encontram a medicina, especificamente a psiquiatria e a psicologia, cujos avanços, ainda que bastante reconhecidos, estão longe de alcançar consensos ou unanimidades em termos de diagnóstico em algumas patologias, torna-se mais evidente a necessidade de se buscarem elementos que possam diminuir a possibilidade de falhas diagnósticas e as respectivas consequências.

A psicopatologia é um campo bastante complexo e, na maioria dos casos, nem todas as suas intrincadas relações chegaram a ser identificadas a ponto de diferenciá-las quanto ao surgimento, ao conteúdo, à dinâmica ou quanto ao tratamento. É quando sinais ou sintomas semelhantes se referem a patologias diferentes. Sintomas semelhantes podem indicar duas ou três doenças e pode-se, de início, se chegar a mais de um diagnóstico, até que se conclua pelo definitivo. Isso não vai significar erro, mas chega a conclusões intermediárias nas etapas de investigação de um mesmo quadro.

A tecnologia pode auxiliar a confirmação do diagnóstico, já que o médico trabalha com sistemas de reconhecimento, baseando-se em suposições daquilo que é estatisticamente provável em uma determinada situação. Mesmo assim, nem sempre um diagnóstico se confirma, sendo necessário voltar ao início e rever os dados para mudar o rumo da análise, para que o tratamento dê resultado ou algum exame confirme aquilo que o médico suspeitava. Isso significa que o diagnóstico de patologias complexas requer do médico, exames completos que representem uma profunda investigação. Além disso, não pode haver atitudes fechadas, como se ater a sintomas e sinais mais evidentes, mais difundidos, desprezando aspectos, como, questões relacionadas à história do paciente.

No caso da esquizofrenia e do TDI, por serem transtornos crônicos, cujo diagnóstico pode ser equivocado, devido às características similares que apresentam, avaliações multidisciplinares são indicadas para se fazer o diagnóstico diferencial entre eles. Julgar que se trata de um ou de outro transtorno apenas por sinais e sintomas, como tende a acontecer na prática clínica da esquizofrenia, pode facilmente esbarrar na areia móvel das transformações inesperadas, que muitas vezes revelam resultados e consequências equivocados.

\section{CONCLUSÕES}

No âmbito da psicologia, o teste de Rorschach tem seu uso referendado como metodologia segura, inclusive no que concerne ao estudo da dissociação patológica da consciência. No âmbito da psiquiatria, esse teste também referenda o diagnóstico, como instrumento peculiar e relevante. Casos em que a Técnica de Rorschach não possa ser aplicada - por falta de condições do paciente para se expressar verbalmente ou por acuidade visual insuficiente - outro instrumento psicológico precisará ser utilizado no psicodiagnóstico, para suprir esta demanda ou carência. Situações dessa natureza não foram contempladas nesta pesquisa. Nesse particular, estudos adicionais e complementares são necessários, uma vez que situações com essas características requerem metodologia heterogênea.

No que diz respeito ao objetivo desta pesquisa, para fins de diagnóstico diferencial, entre os dez pacientes diagnosticados com esquizofrenia investigados, concluise que dois apresentaram sintomas diferenciais aos da esquizofrenia, sendo que um deles apresentava sintomas dissociativos - resposta direta ao objetivo do trabalho. Apesar desse resultado direto, não foi confirmada a prevalência do TDI entre pacientes esquizofrênicos. Mas independentemente do número, o resultado encontrado - um paciente com sintomas dissociativos - confirma a preocupação do pesquisador quanto à existência de diagnósticos equivocados entre os dois transtornos.

Nesse sentido, propõe-se, como forma de solução para se evitar a ocorrência de diagnósticos inadequados para pacientes esquizofrênicos, que seja elaborado, pelas duas áreas prioritariamente envolvidas, a psiquiatria e a psicologia, um protocolo de sinais, sintomas e/ou traços que possam diferenciar a esquizofrenia do TDI. Cabe considerar que, no diagnóstico, tais sintomas e sinais não podem se limitar à identificação de dois pontos fundamentais, o trauma e a dissociação, que praticamente têm sido o foco dos diagnósticos desses dois transtornos. 
$\mathrm{Na}$ esquizofrenia, a suposição de uma base genética e biológica tem um nexo de causalidade Experiências adversas na infância são consideradas irrelevantes, e experiências estressantes ou traumáticas em adulto são vistas como forma de liberação de mecanismos subjacenets da doença. Já o paradigma dominante dos distúrbios dissociativos centra-se quase que exclusivamente em eventos traumáticos ou fatos significativos relacionados com os sintomas que uma pessoa experimenta. Por isso, para o diagnóstico diferencial, tal protocolo precisa ser fruto de uma avaliação psiquiátrica detalhada, paralelamente a um psicodiagnóstico minucioso.

Nesse protocolo, a Técnica de Rorschach pode ser referendada como um instrumento peculiar e relevante. Impreterivelmente, o fator dissociativo tem que estar presente, e a confluência dos indicadores, bem como o diferencial da dissociação ou do grau da patologia dissociativa, determinará o enquadramento dos pacientes em uma das síndromes abordadas neste estudo ou em nenhuma delas.

\section{REFERÊNCIAS}

Amato, D., Beasley, C. L., Hahn, M. K., \& Vernon, A. C. (2016) Neuroadaptations to antipsychotic drugs: Insights from preclinical and human post-mortem studies. Neuroscience and Biobehavioral Reviews, 76(Pt. B), 317-335.

Asher, L., Fekadu, A., \& Hanlon, C. (2018) Global mental health and schizophrenia. Current Opinion in Psychiatry, 31(3), 193-199.

American Psychiatric Association. (2014). Manual diagnóstico $e$ estatístico de transtornos mentais $-5^{a} E d$. . Porto Alegre: Artmed.

Brand, B. L., Loewenstein, R. J., \& Spiegel, D. (2014). Dispelling myths about dissociative identity disorder treatment: an empirically based approach. Psychiatry, 77(2), 169-189.

Braun, B. G. (1990). Multiple personality disorder: An overview. American Journal of Occupational Therapy, 44(11), 978-983.

Carretoni Filho, H., \& Prebianchi, H. B. (1999). Exame clínico psicológico (Anamnese; 2 ed). São Paulo: Casa do Psicólogo.

Cordeiro, Q., Silva, R. T., \& Vallada, H. (2012). Association study between the rs165599 catechol-O-methyltransferase genetic polymorphism and schizophrenia in a Brazilian sample. Arquivos de Neuro-psiquiatria, 70(12), 913-916.

Dell, P. F. (2002). Dissociative phenomenology of dissociative identity disorder. The Journal of Nervous and Mental Disease, 190(1), 10-15.

Dorahy, M. J., Brand, B. L., Sar, V., Krüger, C., Stavropoulos, P., Martínez-Taboas, A., ... Middleton, W. (2014) Dissociative identity disorder: An empirical overview. The Australian and New Zealand Journal of Psychiatry, 48(5), 402-417

Elis, O., Caponigro, J. M., \& Kring, A. M. (2013). Psychosocial treatments for negative symptoms in schizophrenia: Current practices and future directions. Clinical Psychology Review, 33(8), 914-928.

Finsaas, M. C., Bufferd, S. J., Dougherty, L. R., Carlson, G. A., \& Klein, D. N. (2018). Preschool psychiatric disorders: Homotypic and heterotypic continuity through middle childhood and early adolescence. Psychological Medicine, $16,1-10$.

Freud, S. (1996). O Ego e o ID. In. J. Strachey (Ed. e Trans.), Edição STANDARD Brasileira das Obras Psicológicas Completas (Vol. XIX). Rio de Janeiro: Imago. (Trabalho original publicado em 1923)

Gacono, C. B., Loving Jr., J. L., \& Bodholdt, R. H. (2001) The Rorschach and psychopathy: Toward a more accurate understanding of the research findings. Journal of Personality Assessment, 77(1), 16-38

Gentile, J. P., \& Dillon, K. S. (2013). Psychotherapy and pharmacotherapy for patients with dissociative. Innovations in Clinical Neuroscence, 10(2), 22-29.

Gibson, L. E., Alloy, L. B., \& Ellman, L. M. (2016) Trauma and the psychosis spectrum: A review of symptom specificity and explanatory mechanisms. Clinical Psychology Review, 49, 92-105

Haddock, D. B. (2001). Dissociative identity disorder. Sourcebook. New York: McGraw-Hill.
Howes, O. D., McCutcheon, R., Owen, M. J., \& Murray, R. M. (2016). The role of genes, stress, and dopamine in the development of schizophrenia. Biological Psychiatry, 81(1), 9-20.

Kar, N., Barreto, S., \& Chandavarkar, R. (2016) Clozapine monitoring in clinical practice: Beyond the mandatory requirement. Clinical Psychopharmacology and Neuroscience, 14(4), 323-329

Kendler, K. S. (2016) phenomenology of schizophrenia and the representativeness of modern diagnostic criteria. JAMA Psychiatry, 73(10), 1082-1092

Kihlstrom, J. F., Glisky, M. L., \& Angiulo, M. J. (1994). Dissociative tendencies and dissociative disorders. Journal of Abnormal Psychology, 103(1), 117-124.

Kluft, R. P. (1991). Clinical presentations of multiple personality disorder. Psychiatric Clinics of North America, 14(3), 605-629.

Kluft, R. P. (2000). The psychoanalytic psychotherapy of Dissociative Identity Disorder in the context of trauma therapy. Psychoanalytic Inquiry. 20, 259-86.

Kumar, H. B. K., Castellani, C., Maiti, S., O’Reilly, R., \& Singh, S. M. (2013). Search for missing schizophrenia genes will require a new developmental neurogenomic perspective. Journal of Genetics, 92(2), 335-340.

Lakshmanan, M. N., Meier, S. L. C., Meier, R. S., \& Lakshmanan, R. (2010). An archetype of the collaborative efforts of psychotherapy and psychophar macology in successfully treating dissociative identity disorder with comorbid bipolar disorder. Psychiatry, 7(7), 33-37.

Leonard, D., \& Tiller, J. (2016). Dissociative identity disorder (DID) in clinical practice - What you don't see may hurt you. Australas Psychiatry, 24(1), 39-41.

Lewis, D. O., Yeager, C. A., Swica, Y., Pincus, J. H., Lewis, M., \& Psych, F. R. C. (1997). Objective documentation of child abuse and dissociation in 12 murderers with dissociative identity disorder. The American Journal of Psychiatry, 154(12), 1703-1710.

Ma, C., Gu, C., Huo, Y., Li, X., \& Luo, X.J. (2018). The integrated landscape of causal genes and pathways in schizophrenia. Translational Psychiatry, 8(1), 67

Maramai, S., Gemma, S., Brogi, S., Campiani, G., Butini, S., Stark, H., \& Brindisi, M. (2016) dopamine d3 receptor antagonists as potential therapeutics for the treatment of neurological diseases. Frontiers in Neuroscience, 10, 451.

Mihura, J. L., Bombel, G., Dumitrascu, N., Roy, M., \& Meadows, E. A. (2018). why we need a formal systematic approach to validating psychological tests: The case of the Rorschach Comprehensive System. Journal of Personality Assessment, 3, 1-19.

Moskowitz, A. (2011). Schizophrenia, trauma, dissociation, and scientific revolutions. Journal of Trauma \& Dissociation, 12, 347-357.

Negro Júnior, P. J., Palladino-Negro, P., \& Louzã, M. R. (1999). Dissociação e transtornos dissociativos: Modelos teóricos. Revista Brasileira de Psiquiatria, 21(4), 1-24. 
Organização Mundial da Saúde. (1993). Classificação de transtornos mentais e de comportamento da CID-10. Descrições Clínicas e Diretrizes Diagnósticas. Porto Alegre: Artes Médicas.

Putnam, F. W. (1989). Diagnosis and treatment of multiple personality disorder. New York: Guilford.

Putnam, F. W. (1992). Manual for the dissociative experiences scale. New Yok: National Institute of Mental Health.

Ribáry, G., Lajtai, L., Demetrovics, Z., \& Maraz, A. (2017). Multiplicity: An explorative interview study on personal experiences of people with multiple selves. Frontiers in Psychology, 13, 8, 938.

Rich, R. (2005). Got Parts? An insider's guide to managing life successfully with Dissociative Identity Disorder. New York: Loving Healing Press.

Salamon, S., Santelmann, H., Franklin, J., \& Baethge, C. (2018). Test-retest reliability of the diagnosis of schizoaffective disorder in childhood and adolescence - A systematic review and meta-analysis. Journal of Affective Disorders, 230, 28-33.

Schimmenti, A. (2017). Elena: A case of dissociative identity disorder from the 1920s. Bulletin of the Menninger Clinic, 81(3), 281-298.

Schmitt, A., Reich-Erkelenz, D., Gebicke-Härter, P., \& Falkai, P. (2012). Transcriptome studies in the context of disturbed connectivity in schizophrenia. Revista de Psiquiatria Clínica, 40(1), 10-15.

Sims, A. (1988). Symptoms in the mind. London: Ballière Tindall.

Van der Hart, O. (2012). The use of imagery in phase 1 treatment of clients with complex dissociative disorders. European Journal of Psychotraumatology, 3, 10.3402/ejpt.v3i0.8458.

Vaz, C. E. (1997). O Rorschard. Teoria e Desempenho (3 $3^{\mathrm{a}} \mathrm{ed}$.). São Paulo: Manole.

Vila, D., \& Lunazzi, H. (2010). Especificidad de la respuesta contaminada en Rorschach. II Congreso Internacional de Investigación y Práctica Profesional en Psicología XVII. Jornadas de Investigación Sexto Encuentro de Investigadores en Psicología del MERCOSUR. Buenos Aires. Recuperado de www.aacademica.org/000-031/928.pdf

Vita, A., \& Barlati, S. (2018) Recovery from schizophrenia: is it possible? Current Opinion in Psychiatry, 31(3), 246-255

Yu, J., Ross, C. A., Keyes, B. B., Li, Y., Dai. Y., Zhang, T., ... Xiao, Z. (2010). Dissociative disorders among chinese inpatients diagnosed with schizophrenia. Journal of Trauma \& Dissociation, 11, 358-372.

Zimerman, D. E. (1999). Fundamentos Psicanalíticos. São Paulo: Artmed. 